\title{
Interpretation of Neuroimaging Data Based on Network Concepts
}

\author{
Anthony R. McIntosh • Michèle Korostil
}

Received: 10 May 2008 / Accepted: 22 July 2008 / Published online: 14 August 2008

(C) The Author(s) 2008

\begin{abstract}
By capturing the actions of distributed brain regions, neuroimaging can give unique insights into the networks underlying complex behavioral and cognitive functions. An approach to interpreting neuroimaging data grounded in emerging ideas in brain network theory is needed to better characterize these large-scale network dynamics. This paper focuses on three concepts germane to this approach to interpretation: "connectivity", "neural context", and "small-world properties". Measures of brain connectivity emphasize the combined action of areas. Functional connectivity analyses focus on interacting neural patterns, whereas effective connectivity analyses uncover directional influences between brain areas. The second concept, neural context, purports that a region's contribution to a function is more fully appreciated in relation to other coactive brain areas. The final concept is the extension of graph theory measures to the estimation of small-world properties. Measures such as clustering and path length can be used to infer the computational capacity of functional networks. These three constructs are central to the interpretation of neuroimaging data that will further unravel how brain network dynamics guide mental function, and are beginning to be applied to the study of neural disorders.
\end{abstract}

A. R. McIntosh $(\triangle) \cdot$ M. Korostil

Rotman Research Institute of Baycrest Centre,

University of Toronto, 3560 Bathurst Street,

Toronto, ON M6A 2E1, Canada

e-mail: rmcintosh@rotman-baycrest.on.ca

M. Korostil

Centre for Addiction and Mental Health,

University of Toronto, 250 College St,

Toronto, ON M5S 2S1, Canada
Keywords Functional connectivity · Effective connectivity · Functional magnetic resonance imaging $\cdot$ Neuroimaging . Small-world networks $\cdot$ Schizophrenia $\cdot$ Neural context

\section{Introduction}

The advent of neuroimaging has allowed a comprehensive sampling of brain activity. It provides the means to determine whether certain behavior operations are characterized by distributed operations. Imaging studies have consistently observed that several brain regions are engaged during a given behavior. With the improved spatiotemporal resolution of neuroimaging techniques, it has become increasingly clear that behavioral operations are embodied in patterns of distributed networks.

The prevalent interpretation framework for neuroimaging has focused on regional changes in activity, which makes historical sense in that most neuroscience data has been obtained from electrical measures of either single neurons or neuronal populations. In neuroimaging data, a given region may increase activity in response to a stimulus, but the relevance of this change is complicated by the fact that at the same time, several other regions will show activity changes (increases and decreases). What is needed, then, are concepts that allow for the interpretation of neuroimaging data consistent with the level of measurement, namely those that emphasize large-scale network dynamics.

The purpose of this paper is to review a different set of concepts to tie the interpretation of neuroimaging data to the level of large-scale network dynamics. The first concept, connectivity, reflects the fact that brain areas work together in instantiating a particular mental function. Connectivity in this case is a statement of a functional relation that is built upon an anatomical foundation. The 
second concept is neural context. Given the anatomical connections between brain areas, there are a number of ways in which areas can interact. This connectivity provides the opportunity for similar areas to be engaged in functionally distinct networks. The key point is that the contribution of a particular region to a function is best appreciated in the context of other areas that are engaged. The final concept comes from the observation that brain networks can be characterized based on features of graphs of the connections. Both the structural and functional connections seem to be well characterized from the perspective of small-world networks. Such networks have a unique capacity for information processing. These three concepts provide insights into the brain dynamics that support cognitive function that cannot be appreciated from considering single areas. The present paper is meant to provide a general introduction to these concepts, and to illustrate how their combined use can be used to gain new insights into brain function. We point the interested reader to the cited literature for more information on these concepts (e.g., Horwitz 2003; Jirsa and Mcintosh 2007; Sporns and Zwi 2004).

\section{Connectivity}

The term functional connections (connectivity) has been used to refer to the correlations of activity between neural elements in both electrophysiology (Aertsen et al. 1987) and brain imaging (Friston et al. 1993). To say that two neural elements (neurons or brain regions) have a functional connection is to say that these elements show statistically significant correlated activity without reference to how that correlation is mediated. Effective connectivity is a logical progression from functional connectivity and can be defined as the influence or effect one neural element has on another (Friston et al. 1993). The major difference between functional and effective connectivity is best appreciated from the available levels of inference. Because functional connectivity is an estimate of a correlation between neural elements (either pairwise or multivariate), no inferences on the directionality of influences are possible. Effective connectivity estimation requires the specification of a model wherein directional influences are estimated, thus by definition inferences on directionality can be made.

A simple illustration may help to clarify. Consider two simple networks shown in Fig. 1. Network 1 has three nodes where nodes ' $a$ ' and ' $b$ ' are connected by a mediating node ' $c$ '. Network 2 has a similar configuration save for a direct link between nodes ' $a$ ' and ' $b$ '. Estimation of functional connectivity amongst the three nodes in either network would not be able to differentiate between the configurations of the two networks because of the focus on
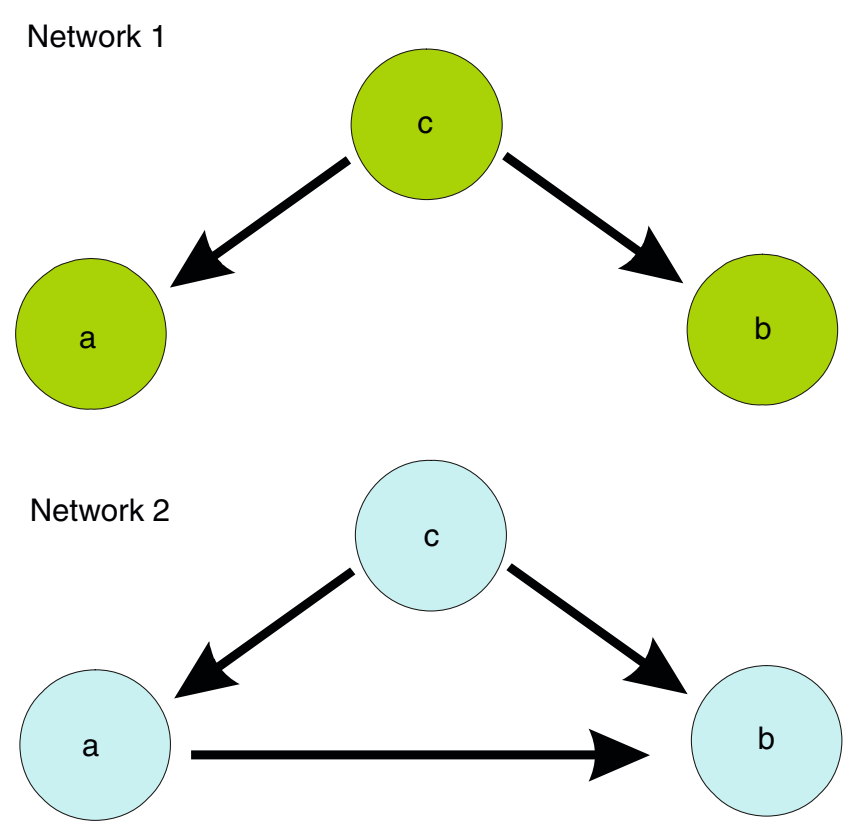

Fig. 1 Example of two networks with different anatomical connection topologies. Functional connectivity estimation between nodes $a, b$, and $c$ in the two networks would not be able to differentiate the anatomical connectivity between networks 1 and 2. Effective connectivity, where directionality of effects are modeled, would be able to identify the direct connection between $a$ and $b$ when the common effect from $c$ is accounted for

zero-order correlations. Effective connectivity, which tries to model directionality, would be able to show that in Network 1, the functional connection between ' $a$ ' and ' $b$ ' is mediated by node ' $c$ ', while in Network 2 , there is an additional influence from ' $a$ ' to ' $b$ ' when the mutual effect from ' $c$ ' is taken into account.

Whether one chooses to estimate one over the other ultimately depends on the nature of the inference one wishes to draw about the data. Specifically, if directionality is important, then effective connectivity needs to be estimated, but if the detection of interacting network nodes is sufficient, then functional connectivity can be estimated (McIntosh and Grady 2007).

It should be pointed out that the term functional connectivity has been applied to both task-dependent changes in functional connections and those that persist in the absence of any overt task, the so-called "resting state functional connectivity" (Biswal et al. 1995). The term functional connectivity is equally valid in both applications, but the emphasis is quite different. In the case of taskdependent functional connectivity, the focus is on whether there is a change in the functional connections between regions as task demands change. Resting-state functional connectivity emphasizes the overall pattern of functional connections and its relation to the underlying anatomy. It is, however, worth remembering the example in Fig. 1, where 
a non-zero functional connection does not guarantee a direct anatomical connection.

Recent modeling work suggests that the patterns of resting-state connectivity are a direct result of the anatomical and functional architecture of the brain (Honey et al. 2007). In a simulated network, as the architecture and dynamics more closely approximate real neural systems, distributed patterns of functionally connected networks emerge. Remarkably, the spatial patterns of these networks resemble those reported in fMRI experiments. If the anatomical structure is perturbed or the dynamics changed, then the patterns break down. Such findings suggest that resting-state connectivity may indeed index the integrity of a given brain. This is substantiated by empirical observations of resting-state correlations in normal aging showing a reduction in overall functional connectivity, particularly among frontal and parietal regions. The reduced functional connectivity correlates with decline in behavioral measures of executive function and overall processing speed (Damoiseaux et al. 2007). Degenerative disorders such as Alzheimer's disease also show reduced functional connectivity related to disease severity (Stam et al. 2006). Taken together, these empirical and modeling findings suggest that resting state correlations may act as a useful "fingerprint" for the integrity of functional networks.

Functional and effective connectivity can also show task-dependent changes. Horwitz et al. (1992) using PET, showed functional connectivity patterns that mapped on to the use of "what" vs. "where" cortical visual pathways. Effective connectivity analyses of these data (McIntosh et al. 1994) showed task-dependent switches in prefrontal feedback, and strong suppressive interactions between "what" and "where" pathways.

Importantly, effective connectivity can differentiate between top-down versus bottom-up effects. Categoryspecific responses have been observed frequently in the ventral occipitotemporal lobe (fusiform gyrus for faces, parahippocampal gyrus for places), which is typically considered a top-down effect from higher-order brain areas. Using a model of effective connectivity, Mechelli et al. (2003) found that early sensory areas changed their effects on category-specific areas in relation to the stimuli, but higher-order association regions did not show such changes in effects. Thus, category specificity in these data was a bottom-up effect where the response was modulated by the effect of lower order cortical regions on category-specific areas. It is likely that the real story of the neural instantiation of category specificity is an outcome of reciprocal interactions amongst neural sites. The results from the effective connectivity analyses enrich models of cognitive function by moving them beyond strict hierarchical representations and emphasizing the dynamic and interactive nature of neural instantiations.
Neural context

Anatomical connectivity enables parallel routes of information flow between areas. Convergent, divergent, and reciprocal connections provide the potential for similar areas to be engaged across different cognitive operations. What distinguishes the operation is not the involvement of the area per se, but rather the status of other areas during that function. This dependence of the contribution of a region on other connected regions has been referred to as "neural context" (Bressler and Mcintosh 2007). Neural context allows the response properties of one element in a network to be profoundly affected by the status of other neural elements in that network. The neural context of each connected area emerges spontaneously from its interactions.

Contextual effects can be demonstrated in simple nervous systems, basic sensory functions, and higher cognitive functions (Bressler and Mcintosh 2007). An appreciation of neural context formed the basis for considering working memory at the confluence of sustained attention and memory. Both working memory and sustained attention involve activity in overlapping regions of PPC, PFC, and anterior cingulate cortex (ACC). In an fMRI study of the relationship between attention and working memory, Lenartowicz and McIntosh (2005) used two variants of a two-back working memory task: a standard version with strong attentional demands, and a cued version that more strongly promoted memory retrieval. The regions functionally connected to the ACC, and the relation of the connectivity patterns to memory performance, differed between tasks. In the standard task, the observed connectivity pattern was related to a speed-accuracy tradeoff, with strong functional connection of ACC to PFC and PPC. In the cued task, the connectivity pattern was related only to better accuracy, and involved functional connections with middle and inferior PFC, and inferior temporal cortex. By virtue of these different patterns of functional connectivity, the contribution of ACC to attention- and memory-driven performance was similarly changed. In other words, each task invoked a different neural context within which the ACC interacted, resulting in two very different behavioral profiles.

In another study of ACC functional connectivity (Stephan et al. 2003), the question was examined of whether hemispheric functional asymmetry was determined by a word stimulus (short words, with one letter colored red) itself or by the task. In one instance, subjects judged whether the word contained the letter " $A$ ", ignoring the red letter, and in another instance, they made a visuospatial judgment indicating whether the red letter was right or left of center. A direct comparison of the activity (measured with fMRI) revealed strong hemispheric differences. The letter task produced higher activity in the left hemisphere, whereas the visuospatial task produced higher activity in 
the right hemisphere. The ACC was similarly active in both tasks relative to baseline, but showed distinctly different patterns of connectivity between tasks. Specifically, during the letter task, the ACC was coupled to the left PFC; during the visuospatial task, the ACC was linked with the right PPC. These data are compelling examples of how behavioral context (in this case, task demands) can modulate the neural context within which a cortical area (i.e., the ACC) operates.

\section{Small-world properties}

Functional connectivity and neural context speak to the dynamics of large-scale networks. Another concept that complements these functional relationships is the assessment of the capacity of a given network to integrate information. This capacity is a hallmark of a complex system, and can be appreciated through characterization of the structural properties of a network.

To function optimally, a complex system such as the brain must balance competing computational demands. It must strike a balance between segregation of modular processes and integration across processes. Additionally, it should be efficient and resilient in the face of perturbation. Evidence is mounting that suggests that the so-called smallworld properties, seen in a wide variety of social, economic, and biological networks, may also underlie the brain's capacity to meet these competing demands.

Watts and Strogatz, in their seminal paper, revealed that widely different sets of natural and artificial networks showed similar small-world attributes (Watts and Strogatz 1998). In a typical small-world network, a large number of connections form densely connected clusters while the remaining connections link the clusters. This feature can be measured to show that small-world systems maintain a balance between high clustering and low path length. Regular, or lattice, networks tend to have high clustering and high path length, whereas random networks have low clustering and low path length. Systems with high clustering will also have a high capacity for segregation, whereas path length between clusters will influence the system's ability to integrate information (Sporns et al. 2000; Tononi et al. 1999). The minimum path length between two nodes is the minimum number of edges that must be traversed to get from one node to the other. Each node in a network can be characterized by the number of edges connected to it, which is referred to as "degree". The larger the degree, the more connected a node. With this measure, "hubs" can be detected as nodes having a higher degree than the average degree.

Graph theory analyses of anatomical connectivity have demonstrated small-world architecture in nervous systems ranging from $c$ elegans through primates (Sporns and Zwi 2004). Functional connectivity measures can also be used to construct graphs that can be assessed for small-world properties. Stam and de Bruin (2004) reported the first graph theoretical analysis of MEG data in five healthy subjects in a resting state by thresholding functional connectivity matrices to create undirected graphs specific to each frequency band. Graphs from alpha and beta band had lattice-like topology, but those from the high and low frequency bands demonstrated small-world properties. Although the temporal resolution of fMRI is far below that of MEG, small-world architecture has been measured using correlations of low-frequency BOLD signals in either the temporal or the spectral domain. Salvador et al. (2005) calculated functional connectivity between 90 anatomically derived regions of interest measured with fMRI. Multivariate analysis by hierarchical clustering and multidimensional scaling consistently defined six major systems in healthy volunteers: medial temporal, subcortical, occipital, frontal, temporal, and sensorimotor. Using a wavelet-based functional connectivity analysis, Achard et al. (2006) showed small-world properties of fMRI networks across several frequencies. The degree distribution across regions followed an exponentially truncated power law, indicating the presence of hubs. These hubs were predominately heteromodal and unimodal association cortices. Unimodal association cortices had highly clustered mostly short-range connections, whereas heteromodal cortices had less clustered, more long-range connections.

Integration of measures and clinical extensions

Network concepts provide the means to move the focus of neuroimaging interpretation from single brain areas to integrated systems. Notions such as "synchrony", "topdown", "bottom-up", and "modulatory" effects, are often invoked in the interpretation of regional changes, but are best accessed by examining connectivity. Neural contextual effects aid in the interpretation of situations where areas typically not associated with a given functional network can nevertheless show strong participation. A region can contribute to a function, yet not be critical for its normal expression.

Contextual dependency may change in different populations due to factors such as maturation, learning, brain damage, or disease. In such cases, evaluation of changes in neural context may give valuable information on how a network reconfigures and whether certain reconfigurations are more successful than others. Characterization of smallworld properties of brain networks provides complementary information by identifying whether regions that show contextual modulation also have unique graph metrics. For example, it may be that hubs are most likely to show contextual modulation whereas regions that are central to clusters show less contextual dependency. Hubs also have a unique capacity for facilitating information integration. Recent work (Honey and Sporns 2008) shows that damage 
to hub nodes in large-scale network simulations has the most dramatic effect on the integrative capacity of the remaining network, particularly when prefrontal (area 46 and FEF) or parietal (areas 5 and 7) cortices are lesioned. Merging such modeling work, with estimation of network operations in empirical data, will enable us to begin to understand why damage to certain areas causes a deficit.

Network concepts have probably been most extensively applied to the study of schizophrenia. In this case, the hypothesis of schizophrenia as a dysconnection syndrome is particularly relevant (Friston and Frith 1995). Initial examinations of functional connectivity generally revealed reduced connectivity in patient populations relative to controls (McGuire and Frith 1996). However, more detailed studies of effective connections indicated that for a given network, one could identify equivalent connections, weaker connections, and connections that were stronger in patients (Jennings et al. 1998), both related and unrelated to treatment (Schlosser et al. 2003). Small-world characterization of the network capacity in schizophrenia has shown reduced small-world topologies, particularly for prefrontal and parietal cortices (Liu et al. 2008). Interestingly, these same areas are identified as showing group differences in studies of effective connectivity (Jennings et al. 1998; Schlosser et al. 2003), and, as noted above, prefrontal and parietal damage compromises the integrative capacity of cortical networks (Honey and Sporns 2008).

Combined with the connectivity estimation, a picture emerges wherein dysconnectivity has both global and local impact that can be related to the expression of the disorder. At a local level, prefrontal cortex is often implicated as central to understanding the disorder (Weinberger et al. 1994), but assessment at the network level demonstrates that prefrontal abnormalities have a broader impact on the overall network dynamics. Indeed, it may be possible that regional deficiencies are an expression of the state of the network. Network analyses agree that there is overlap in the network configurations of patients and controls. Conventional statistical analyses tend to emphasize differences, losing sight that these focal differences are part of a larger network, where some constituents are the same as controls. The change in regional involvement, whether in absolute terms or in degree, would thus translate to a change in neural context. The contextual changes would produce neural dynamics whose emergent function is quite distinct from controls. Studies of large-scale nonlinear interactions in patients suggest that the overall capacity for information integration may not differ substantially between patients and controls, but rather the areas that are integrated in a given functional network (Rubinov et al. 2007). Considered in another way, it may not always be the case that the quantity of information represented in a network is compromised, but rather the quality of such information.
Adopting network concepts for the interpretation of neuroimaging data has implications for the development of neurocognitive theory and for the understanding of brain disorders. Neurocognitive theories are often based primarily on behavioural studies. The theories attempt to link cognitive constructs to neural architecture. Characterization of imaging data as networks provides direct means of evaluating how and whether the neural instantiation resembles the cognitive model. The clinical implications come at several levels. Because the brain is a dynamic system, any insult will have local and global effects. Lesions may lead to focal deficits, but it is more likely that the deficit represents a combination of the loss of the region per se as well as the attempt of the remaining network to compensate.

Acknowledgements This work was supported by JS McDonnell Foundation Grant 22002082

Open Access This article is distributed under the terms of the Creative Commons Attribution Noncommercial License which permits any noncommercial use, distribution, and reproduction in any medium, provided the original author(s) and source are credited.

\section{References}

Achard, S., Salvador, R., Whitcher, B., Suckling, J., \& Bullmore, E. (2006). A resilient, low-frequency, small-world human brain functional network with highly connected association cortical hubs. The Journal of Neuroscience, 26(1), 63-72. doi:10.1523/ JNEUROSCI.3874-05.2006.

Aertsen, A., Bonhoeffer, T., \& Kruger, J. (1987). Coherent activity in neuronal populations: analysis and interpretation. In E. R. Caianiello (Ed.), Physics of Cognitive Processes (pp. 1-34). Singapore: World Scientific Publishing.

Biswal, B., Yetkin, F. Z., Haughton, V. M., \& Hyde, J. S. (1995). Functional connectivity in the motor cortex of resting human brain using echo-planar MRI. Magnetic Resonance in Medicine, 34(4), 537-541. doi:10.1002/mrm.1910340409.

Bressler, S. L., \& Mcintosh, A. R. (2007). The role of neural context in large-scale neurocognitive network operations. In V. K. Jirsa \& A. R. McIntosh (Eds.), Handbook of Brain Connectivity (pp. 403-420). Heidelberg: Springer.

Damoiseaux, J. S., Beckmann, C. F., Arigita, E. J., Barkhof, F., Scheltens, P., Stam, C. J., et al. (2007). Reduced resting-state brain activity in the "default network" in normal aging. Cereb Cortex.

Friston, K. J., \& Frith, C. D. (1995). Schizophrenia: a disconnection syndrome? Clinical Neuroscience (New York, N.Y.), 3(2), 89-97.

Friston, K., Frith, C., \& Fracowiak, R. (1993). Time-dependent changes in effective connectivity measured with PET. Human Brain Mapping, 1, 69-79. doi:10.1002/hbm.460010108.

Honey, C. J., Kotter, R., Breakspear, M., \& Sporns, O. (2007). Network structure of cerebral cortex shapes functional connectivity on multiple time scales. Proceedings of the National Academy of Sciences of the United States of America, 104(24), 10240-10245. doi:10.1073/pnas.0701519104.

Honey, C. J., \& Sporns, O. (2008). Dynamical consequences of lesions in cortical networks. Hum Brain Mapp. 
Horwitz, B. (2003). The elusive concept of brain connectivity. NeuroImage, 19 (2 Pt 1), 466-470. doi:10.1016/S1053-8119(03) 00112-5.

Horwitz, B., Grady, C. L., Haxby, J. V., Schapiro, M. B., Rapoport, S. I., Ungerleider, L. G., et al. (1992). Functional associations among human posterior extrastriate brain regions during object and spatial vision. Journal of Cognitive Neuroscience, 4(4), 311322. doi:10.1162/jocn.1992.4.4.311.

Jennings, J. M., McIntosh, A. R., Kapur, S., Zipursky, R. B., \& Houle, S. (1998). Functional network differences in schizophrenia: A rCBF study of semantic processing. Neuroreport, 9(8), 16971700. doi:10.1097/00001756-199806010-00005.

Jirsa, V. K., \& Mcintosh, A. R. (Eds.) (2007). Handbook of Brain Connectivity. Heidelberg: Springer.

Lenartowicz, A., \& McIntosh, A. R. (2005). The Role of Anterior Cingulate Cortex in Working Memory is Shaped by Functional Connectivity. Journal of Cognitive Neuroscience, 17(7), 10261042. doi:10.1162/0898929054475127.

Liu, Y., Liang, M., Zhou, Y., He, Y., Hao, Y., Song, M., et al. (2008). Disrupted small-world networks in schizophrenia. Brain, $131(\mathrm{Pt}$ 4), 945-961. doi:10.1093/brain/awn018.

McGuire, P. K., \& Frith, C. D. (1996). Disordered functional connectivity in schizophrenia. Psychological Medicine, 26(4), 663-667.

McIntosh, A. R., \& Grady, C. L. (2007). Network analysis of the human brain: Applications to understanding normal and abnormal neural system operations. In F. G. Hillary \& J. Deluca (Eds.), Functional neuroimaging in clinical populations (pp. 117-144). New York: The Guilford Press.

McIntosh, A. R., Grady, C. L., Ungerleider, L. G., Haxby, J. V., Rapoport, S. I., \& Horwitz, B. (1994). Network analysis of cortical visual pathways mapped with PET. The Journal of Neuroscience, 14, 655-666.

Mechelli, A., Price, C. J., Noppeney, U., \& Friston, K. J. (2003). A dynamic causal modeling study on category effects: bottom-up or top-down mediation? Journal of Cognitive Neuroscience, 15(7), 925-934. doi:10.1162/089892903770007317.

Rubinov, M., Knock, S. A., Stam, C. J., Micheloyannis, S., Harris, A. W., Williams, L. M., et al. (2007). Small-world properties of nonlinear brain activity in schizophrenia. Hum Brain Mapp.
Salvador, R., Suckling, J., Coleman, M. R., Pickard, J. D., Menon, D., \& Bullmore, E. (2005). Neurophysiological architecture of functional magnetic resonance images of human brain. Cerebral Cortex (New York, N.Y.), 15(9), 1332-1342. doi:10.1093/cercor/ bhi016.

Schlosser, R., Gesierich, T., Kaufmann, B., Vucurevic, G., Hunsche, S., Gawehn, J., et al. (2003). Altered effective connectivity during working memory performance in schizophrenia: a study with fMRI and structural equation modeling. NeuroImage, 19(3), 751763. doi:10.1016/S1053-8119(03)00106-X.

Sporns, O., Tononi, G., \& Edelman, G. M. (2000). Connectivity and complexity: the relationship between neuroanatomy and brain dynamics. Neural Networks, 13(8-9), 909-922. doi:10.1016/ S0893-6080(00)00053-8.

Sporns, O., \& Zwi, J. D. (2004). The small world of the cerebral cortex. Neuroinformatics, 2(2), 145-162. doi:10.1385/ $\mathrm{NI}: 2: 2: 145$.

Stam, C. J., \& de Bruin, E. A. (2004). Scale-free dynamics of global functional connectivity in the human brain. Human Brain Mapping, 22(2), 97-109. doi:10.1002/hbm.20016.

Stam, C. J., Jones, B. F., Manshanden, I., van Cappellen van Walsum, A. M., Montez, T., Verbunt, J. P., et al. (2006). Magnetoencephalographic evaluation of resting-state functional connectivity in Alzheimer's disease. NeuroImage, 32(3), 1335-1344. doi:10.1016/j.neuroimage.2006.05.033.

Stephan, K. E., Marshall, J. C., Friston, K. J., Rowe, J. B., Ritzl, A., Zilles, K., et al. (2003). Lateralized cognitive processes and lateralized task control in the human brain. Science, 301(5631), 384-386. doi:10.1126/science.1086025.

Tononi, G., Sporns, O., \& Edelman, G. M. (1999). Measures of degeneracy and redundancy in biological networks. Proceedings of the National Academy of Sciences of the United States of America, 96(6), 3257-3262. doi:10.1073/pnas.96.6.3257.

Watts, D. J., \& Strogatz, S. H. (1998). Collective dynamics of 'smallworld' networks. Nature, 393(6684), 440-442. doi:10.1038/ 30918.

Weinberger, D. R., Aloia, M. S., Goldberg, T. E., \& Berman, K. F. (1994). The frontal lobes and schizophrenia. The Journal of Neuropsychiatry and Clinical Neurosciences, 6(4), 419-427. 